\title{
Triangles in Euclidean Arrangements
}

\author{
S. Felsner and K. Kriegel \\ Fachbereich Mathematik und Informatik, Freie Universität Berlin, \\ Takustrasse 9, 14195 Berlin, Germany \\ \{felsner,kriegel\}@inf.fu-berlin.de
}

\begin{abstract}
The number of triangles in arrangements of lines and pseudolines has been the object of some research. Most results, however, concern arrangements in the projective plane. In this article we add results for the number of triangles in Euclidean arrangements of pseudolines. Though the change in the embedding space from projective to Euclidean may seem small there are interesting changes both in the results and in the techniques required for the proofs.

In 1926 Levi proved that a nontrivial arrangement-simple or not-of $n$ pseudolines in the projective plane contains at least $n$ triangles. To show the corresponding result for the Euclidean plane, namely, that a simple arrangement of $n$ pseudolines contains at least $n-2$ triangles, we had to find a completely different proof. On the other hand a nonsimple arrangement of $n$ pseudolines in the Euclidean plane can have as few as $2 n / 3$ triangles and this bound is best possible. We also discuss the maximal possible number of triangles and some extensions.
\end{abstract}

\section{Introduction, Definitions, and Overview}

The number $p_{3}$ of triangles in arrangements of (pseudo)lines has been the object of previous research. In this article we add new results concerning the number of triangles in Euclidean arrangements of pseudolines.

Grünbaum [Gr] defines an arrangement $\mathcal{A}$ of lines as a finite collection $\left\{L_{0}, L_{1}, \ldots, L_{n}\right\}$ of lines, i.e., one-dimensional subspaces in the real projective plane $\mathbb{P}$. Specifying a line $L_{0}$ in $\mathcal{A}$ as the "line at infinity" induces the arrangement $\mathcal{A}_{L_{0}}$ of lines $\left\{L_{1}, \ldots, L_{n}\right\}$ in the Euclidean plane $\mathbb{E}=\mathbb{P} \backslash L_{0}$.

With an arrangement we associate the cell complex of vertices, edges, and cells into which the lines of the arrangement decompose the underlying space $\mathbb{P}$ or $\mathbb{E}$. Arrangements are isomorphic provided their cell complexes are isomorphic. 


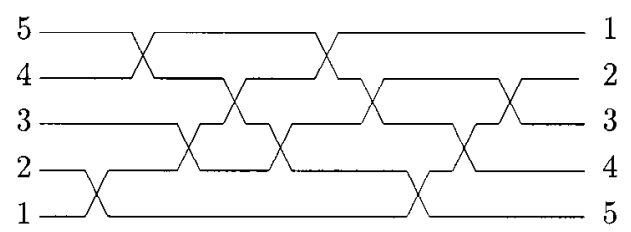

Fig. 1. Wiring diagram of a simple arrangement of five pseudolines.

An arrangement $\mathcal{B}$ of pseudolines in $\mathbb{P}$ is a collection $\left\{P_{0}, P_{1}, \ldots, P_{n}\right\}$ of simple closed curves (we call them pseudolines) in $\mathbb{P}$ such that every two curves have exactly one point in common. Specifying a pseudoline $P_{0}$ in $\mathcal{B}$ as the line at infinity induces the arrangement $\mathcal{B}_{P_{0}}$ of pseudolines $\left\{P_{1}, \ldots, P_{n}\right\}$ in $\mathbb{P} \backslash P_{0}$. Since $\mathbb{P} \backslash P_{0}$ is homeomorphic to the Euclidean plane and we are interested in properties of the induced cell complex we may regard $\mathcal{B}_{P_{0}}$ as an arrangement in $\mathbb{E}$.

In the early work by Levi [Le] and Ringel [Ri] it had already been noted that arrangements of pseudolines are a proper generalization of arrangements of lines. This is due to the existence of incidence laws in plane geometry, e.g., the Theorem of Pappus. Arrangements of pseudolines have received attention since they provide a generic model for oriented matroids of rank 3. In this context questions of strechability have attained considerable interest. For more about these connections we refer the reader to the "bible of oriented matroids" $\left[\mathrm{BLS}^{+}\right]$.

An arrangement is called trivial if all the (pseudo)lines intersect in a single point. If no point belongs to more then two of the (pseudo)lines we call the arrangement simple.

Euclidean arrangements of pseudolines are the main object of investigations in this paper. Work with these objects is simplified by the fact that every arrangement of pseudolines, i.e., of doubly unbounded curves, is isomorphic to an arrangement of $x$-monotone pseudolines, i.e., of curves that intersect every vertical line in exactly one point. Particularly nice pictures of Euclidean arrangements of pseudolines are given by their wiring diagrams introduced by Goodman [Go], see Fig. 1. In this representation the $n x$-monotone curves are restricted to $n y$-coordinates except for some local switches where adjacent lines cross. Knuth $[\mathrm{Kn}]$ points out a connection with "primitive sorting networks." We now summarize bounds for the number $p_{3}$ of triangles in arrangements.

Theorem 1. For every nontrivial arrangement $\mathcal{A}$ of $n$ pseudolines in $\mathbb{P}$ :

(1) Every pseudoline is incident with at least three triangles. Since every triangle is incident with three lines this implies $p_{3}(\mathcal{A}) \geq n$.

(2) $p_{3}(\mathcal{A}) \leq \frac{1}{3} n(n-1)$ for $n \geq 9$. Equality holds for some arrangements of $n$ pseudolines for infinitely many values of $n$.

Part (1) is due to Levi [Le]. The lower bound for $p_{3}$ it best possible. To see this take the $n$ supporting lines of the edges of a regular $n$-gon for $n \geq 4$. The arrangement thus obtained is a simple arrangement of lines with $p_{3}=n$.

Part(2) has a more entangled history. In [Gr] the following easy argument for $p_{3} \leq$ $\frac{1}{3} n(n-1)$ in simple arrangements is given: Since $\mathcal{A}$ is simple only one of the cells bounded by an edge can be a triangle. There are $n(n-1)$ edges and every triangle 
uses three of them. This proves the bound. Grünbaum conjectured the same bound for nonsimple arrangements of lines with sufficiently large $n$. Several lower bounds and special cases were proved by Strommer [St], Purdy [Pu1], [Pu2], and Entringer and Purdy [EP]. Finally, Roudneff [Rou3] proved the conjecture for $n \geq 9$. By perturbing high degree vertices so that suitable arrangements are formed in the neighborhood he shows that $p_{3}$ is maximized by what he calls "reduced arrangements." In particular these arrangements have no vertices of degree more than four. The crucial part of the proof is to show that if $t_{i}$ counts vertices of degree $i$, then for $n \geq 9$ every reduced arrangement has

$$
3 p_{3} \leq 2\left(t_{2}+3 t_{3}+6 t_{4}\right) \text {. }
$$

Since $\sum_{k}\left(\begin{array}{l}k \\ 2\end{array}\right) t_{k}=\left(\begin{array}{l}n \\ 2\end{array}\right)$ this implies the bound.

Infinite families of simple arrangements with $p_{3}=\frac{1}{3} n(n-1)$ have been obtained by Roudneff [Rou1] and Harborth [Ha]. For stretchable arrangements the best known constructions are due to Füredi and Palásti [FP]. Their examples have at least $\frac{1}{3} n(n-3)$ triangles. Forge and Ramírez-Alfonsín [FR] construct simple stretchable arrangements with $p_{3}=\frac{1}{3} n(n-1)$.

In this paper we discuss triangles in Euclidean arrangements. The cell complex of an arrangement in $\mathbb{E}$ consists of unbounded and bounded cells. In our treatment we ignore unbounded cells. In the arrangement of Fig. 1 we thus count three triangles and three quadrangles. Our main results are summarized in the following theorem whose proof is given in Sections 2 and 3.

\section{Theorem 2. For every nontrivial arrangement $\mathcal{B}$ of $n$ pseudolines in $\mathbb{E}$ :}

(1) If $\mathcal{B}$ is simple, then $p_{3}(\mathcal{B}) \geq n-2$. Equality is possible for all $n \geq 3$.

(2) If $n \geq 6$, then $p_{3}(\mathcal{B}) \geq \frac{2}{3} n$. Equality is possible for all $n=0 \quad(\bmod 3)$.

(3) $p_{3}(\mathcal{B}) \leq \frac{1}{3} n(n-2)$. Equality is possible for infinitely many values of $n$.

Part (1) again has a long history. In 1889 Roberts [Rob] claimed that every simple arrangement $\mathcal{A}$ of $n$ lines in $\mathbb{E}$ contains $n-2$ triangles. The argument however was flawed. Ninety years later Shannon [Sh] proved Robertss' theorem using dual configurations. Actually, he proved the analog of Robertss' theorem for arbitrary dimensions: every arrangement of $n$ hyperplanes in $\mathbb{R}^{d}$ contains at least $n-d$ simplicial $d$-cells. Shannon's proof does not require that the arrangement is simple. Therefore, Shannon's theorem together with Theorem 2(2) gives the following amazing result.

Corollary 3. If $p_{3}(\mathcal{B})<n-2$ for an arrangement $\mathcal{B}$ of $n$ pseudolines, then $\mathcal{B}$ is nonstrechable.

A similar effect in the projective setting was conjectured by Grünbaum and proved by Roudneff [Rou2]. A nonsimple projective arrangement with $p_{3}=n$ is nonstrechable. An example of such an arrangement is due to Canham, see p. 55 of [Gr]. In Section 3 we describe a family $W_{n}$ of arrangements with few triangles. If $W_{n}$ is considered as an arrangement in the projective plane it is a nonsimple arrangement with $n$ lines and $p_{3}=n$.

It is interesting to note that Levi's theorem about the number of triangles incident to a line in a projective arrangement and Theorem 2(1) about the number of triangles in 
Euclidean arrangements both give easy double-counting proofs for the bound $p_{3} \geq n$ in the projective case. We elaborate the second:

Corollary 4. The number of triangles in a simple arrangement $\mathcal{A}$ of $n$ pseudolines in $\mathbb{P}$ is at least $n$.

Proof. For each pseudoline $P_{i}$ consider the Euclidean arrangement $\mathcal{A}_{P_{i}}$ obtained by taking $P_{i}$ as line at infinity. Each such arrangement has at least $(n-1)-2$ triangles. Altogether this gives at least $n(n-3)$ triangles. Any fixed triangle $\Delta$ in $\mathcal{A}$ is bounded by three pseudolines and hence counted exactly $n-3$ times. This shows that there are at least $n$ different triangles.

The upper bound on the number of triangles in the Euclidean case claimed in (3) of Theorem 2 can be proved along the lines of Roudneff's upper bound for the projective case. The proof is long and the changes necessary to adapt it to the Euclidean case are obvious. Therefore, we refrain from elaborating on it and refer to Roudneff's original paper [Rou3].

To show that the bound is best possible again the examples from the same paper [Rou3] do the work. Roudneff shows that there is an infinite family of simple projective arrangements with $n+1$ lines and $(n+1) n / 3$ triangles. Each line of such an arrangement is incident to $n$ triangles. Choose an arbitrary line $l$ as a line in infinity. The remaining Euclidean arrangement of $n$ lines has $(n+1) n / 3-n=n(n-2) / 3$ triangles.

\section{Simple Euclidean Arrangements}

In this section we prove the lower bound for the number of triangles in simple arrangements of pseudolines in $\mathbb{E}$. For arrangements of lines (even nonsimple ones) the same bound has been obtained by Shannon [Sh] using dual configurations in $(n-2)$ dimensional space. Our argument is confined to considerations in two dimensions.

Proposition 2.1. $\quad p_{3}(\mathcal{B}) \geq n-2$ for every simple arrangement $\mathcal{B}$ of $n$ pseudolines in $\mathbb{E}$.

Proof. We consider the finite part of $\mathcal{B}$ as a planar graph. Let $V$ be the number of vertices, let $E$ be the number of edges, and let $F$ be the number of (bounded!) faces. These statistics can all be expressed as functions of the number of pseudolines.

$$
V=\left(\begin{array}{l}
n \\
2
\end{array}\right), \quad E=n(n-2), \quad F=\left(\begin{array}{c}
n-1 \\
2
\end{array}\right) .
$$

Note that in this setting Euler's formula gives $V-E+F=1$.

We assign labels $\oplus$ or $\ominus$ to each side of every edge. Let $f$ be one of the two (possibly unbounded) faces bounded by $e$ and let $e^{\prime}$ and $e^{\prime \prime}$ be the edge-neighbors of $e$ along $f$. Let $l, l^{\prime}$, and $l^{\prime \prime}$ be the supporting pseudolines of $e, e^{\prime}$, and $e^{\prime \prime}$, respectively. The label of $e$ on the side of $f$ is $\oplus$ if $f$ is contained in the finite triangle $T$ of the arrangement $\left\{l, l^{\prime}, l^{\prime \prime}\right\}$, 


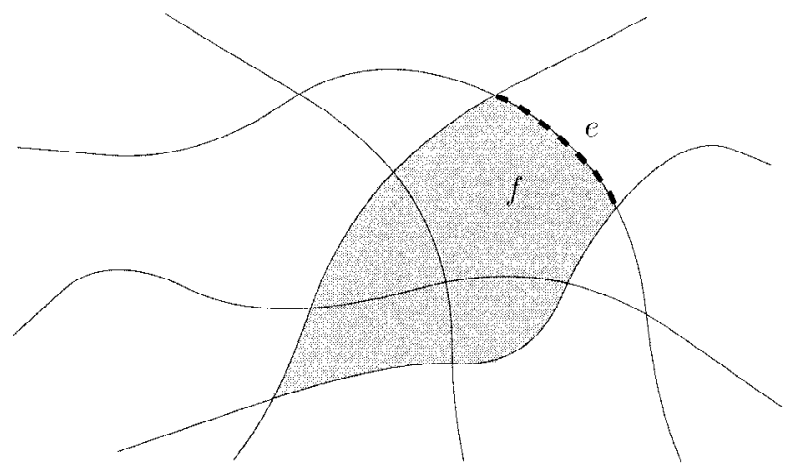

Fig. 2. The label of $e$ at $f$ is $\oplus$ since $f$ is contained in the shaded triangle.

otherwise the label is $\ominus$. See Fig. 2 for an illustration of the definition and Fig. 3 for a complete labeling. With the next lemmas we collect important properties of the edge labeling.

Lemma 5. Every edge e of a simple arrangement has $a \oplus$ and a $\ominus$ label.

Proof. Let $f_{1}$ and $f_{2}$ be the two faces bounded by $e$ and let $e_{1}^{\prime}, e_{1}^{\prime \prime}$ and $e_{2}^{\prime}, e_{2}^{\prime \prime}$ be the edge-neighbors of $e$ in these two faces. Since the arrangement is simple the supporting lines $\left\{l_{1}^{\prime}, l_{1}^{\prime \prime}\right\}$ of both pairs of edges are the same. The finite triangular region $T$ of the arrangement $\left\{l, l^{\prime}, l^{\prime \prime}\right\}$ has edge $e$ on its boundary. Therefore, exactly one of the two faces $f_{1}$ and $f_{2}$ is contained in $T$.

We adopt the notation $T(e)$ for the triangular region $T$ in the arrangement $\left\{l, l^{\prime}, l^{\prime \prime}\right\}$. (In Fig. 2 this is the shaded triangle.)

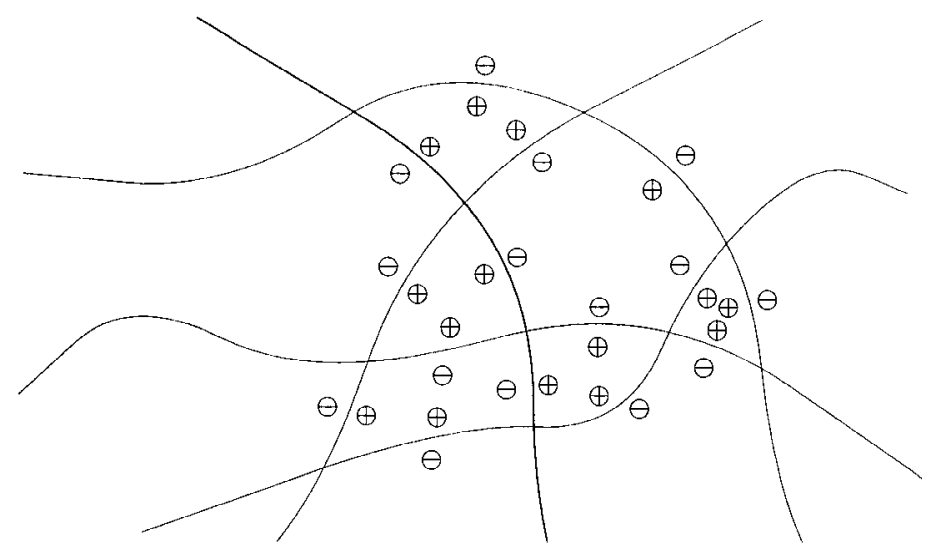

Fig. 3. The arrangement of Fig. 2 with the completed edge labeling. 
Lemma 6. All three edge labels in a triangle are $\oplus$. Every quadrangle contains two $\oplus$ and two $\ominus$ labels. For $k \geq 5$ a $k$-sided face contains at most two $\oplus$ labels.

Proof. If $f$ is a triangle, then for each of its edges $e$ the triangular region $T(e)$ is $f$ itself.

Let $f$ be a quadrangle and let $e, \bar{e}$ be a pair of opposite edges of $f$. Both edges have the same neighboring edges, hence, two of the lines bounding the triangles $T(e)$ and $T(\bar{e})$ are equal. It is easy to see that either $T(e)=f \cup T(\bar{e})$ or $T(\bar{e})=f \cup T(e)$. In the first case $e$ has label $\oplus$ and $\bar{e}$ has label $\ominus$ in $f$, in the second case the labels are exchanged. The second pair of opposite edges also has one label $\oplus$ and the other $\ominus$.

Let $f$ be a face with $k \geq 5$ sides; the lemma immediately follows from the following

Claim. Any two edges with label $\oplus$ in $f$ are neighbors, i.e., share a common vertex.

Let $e_{1}, e_{2}, \ldots, e_{k}$ be the edges of $f$ numbered in counterclockwise direction along $f$ and let $l_{i}$ be the supporting line of $e_{i}$. Let $e_{1}$ have label $\oplus$ and consider an edge $e_{i}$ with $4 \leq i \leq k-2$. We show that the label of $e_{i}$ is $\ominus$ :

Face $f$ is contained in $T\left(e_{1}\right)$ and line $l_{i}$ has to leave $T\left(e_{1}\right) \backslash f$ through $l_{k}$ and $l_{2}$. Figure 4 is a generic sketch of the situation.

Consider line $l_{i-1}$. This line enters the region $R_{1}$ bounded by $l_{2}, l_{i}$, and the chain of edges $e_{3}, e_{4}, \ldots, e_{i-1}$ at the vertex $e_{i-1} \cap e_{i}$. To leave region $R_{1}$ line $l_{i-1}$ has to cross $l_{2}$. Therefore, $l_{i-1}$ has to leave the region $R_{2}$ bounded by $l_{i}, l_{2}$, and $l_{k}$ through $l_{k}$. Symmetrically, $l_{i+1}$ has a crossing with $l_{k}$ to leave the region bounded by $l_{k}, l_{i}$, and the chain of edges $e_{i+1}, e_{i+2}, \ldots, e_{k}$. Therefore, to leave region $R_{2}$ line $l_{i+1}$ has to cross $l_{2}$. This shows that $l_{i-1}$ and $l_{i+1}$ cross inside region $R_{2}$. Hence, $T\left(e_{i}\right)$ is contained in $R_{2}$ and $e_{i}$ has a label $\ominus$ in $f$.

It remains to show that if $e_{1}$ is labeled $\oplus$, then neither $e_{3}$ nor $e_{k-1}$ are. Considering the crossing of lines $l_{4}$ and $l_{2}$ observe that $T\left(e_{3}\right)$ is contained in $T\left(e_{1}\right) \backslash f$. Hence, the label of $e_{3}$ in $f$ is $\ominus$. A symmetric argument applies to $e_{k-1}$. This completes the proof of the claim.

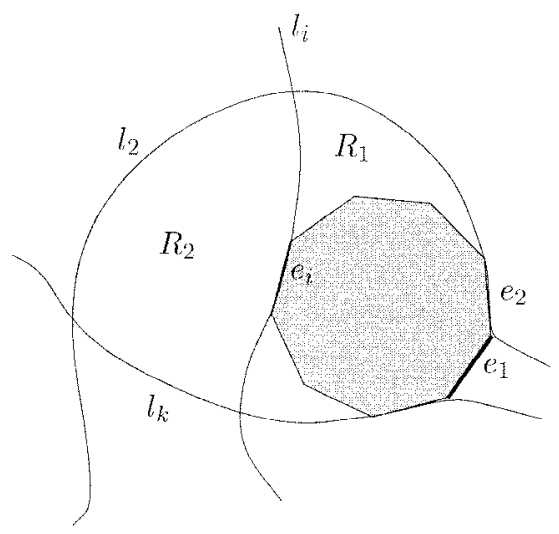

Fig. 4. Edge $e_{1}$ has label $\oplus$ in $f$ so $e_{i}$ must have $\ominus$. 
We use the two lemmas to count the number of $\oplus$ labels in different ways:

$$
E=\sum_{f} \#\{\oplus \text { labels in } f\} \leq 2 F+p_{3} .
$$

With $E=n(n-2)$ and $2 F=(n-1)(n-2)$ this implies

$$
p_{3} \geq n-2 \text {. }
$$

\section{Nonsimple Euclidean Arrangements}

We now come to the lower bound for the number of triangles in the nonsimple case.

Proposition 3.1. A Euclidean nonsimple and nontrivial arrangement of $n \geq 6$ pseudolines has at least $\lceil 2 n / 3\rceil$ triangles, with equality for all $n$.

Proof. We distinguish two cases. First suppose that every line $l$ of the arrangement contains crossings of the arrangement in both open halfspaces it defines. Consider $l$ as a state of a sweepline going across the arrangement. From the theory of sweeps for arrangements of pseudolines (see, e.g., [SH]) we know that the sweep can make progress both in the forward as well as in the backward direction. A progress-move pulls line $l$ across a crossing $c$ of some lines of the arrangement with the property that the parts of all lines contributing to $c$ between $c$ and $l$ are free of further crossings, i.e., are edges of the cell complex induced by the arrangement. Hence, such a move pulls $l$ across some triangles with corner $c$ and an edge on $l$. This shows that $l$ contributes to at least one triangle on either side. Since we assumed that every line has crossings on both sides this accounts for $2 n$ triangles each counted at most three times and the inequality is proved in this case.

Now assume that there is a line $l$ so that all crossings of the arrangement not on $l$ are on one side of $l$. If, on taking away $l$, all lines cross in just one point $c$, then there are $n-2$ triangles in the arrangement and since we assume $n \geq 6$ we are done. Else removing $l$ from the arrangement we still have a nontrivial arrangement which by induction has at least $2(n-1) / 3$ triangles. Since $l$ can make a sweep move to one of its sides there is at least one triangle with an edge on $l$ that disappeared after removal of $l$ (it turned into an unbounded region). This makes a total of $2(n-1) / 3+1>2 n / 3$ triangles in the initial arrangement.

It remains to describe a family $W_{n}$ of arrangements with $3 n$ lines but only $2 n$ triangles. A drawing of $W_{4}$ is given in Fig. 5. It is easy to extend $W_{n}$ by one/two lines such that the number of triangles only increases by one/two.

Let $P$ be a regular $2 n$-gon with edges $e_{1}, e_{2}, \ldots, e_{2 n}$ in counterclockwise ordering and barycenter $c$. Let lines $l_{1}, \ldots, l_{2 n}$ be straight lines such that $l_{i}$ contains edge $e_{i}$ of $P$. Orient the lines such that $P$ is to their left. Note that $l_{i}$ is crossed by lines $l_{i+n+1}, l_{i+n+2}, \ldots, l_{i-1}, l_{i+1}, l_{i+2}, \ldots l_{i+n-1}$ in this order with indices being taken cyclically. The arrangement $\mathcal{A}$ formed by these $2 n$ lines has $2 n$ triangles all adjacent to $P$. All the other faces of the arrangement are quadrangles. 


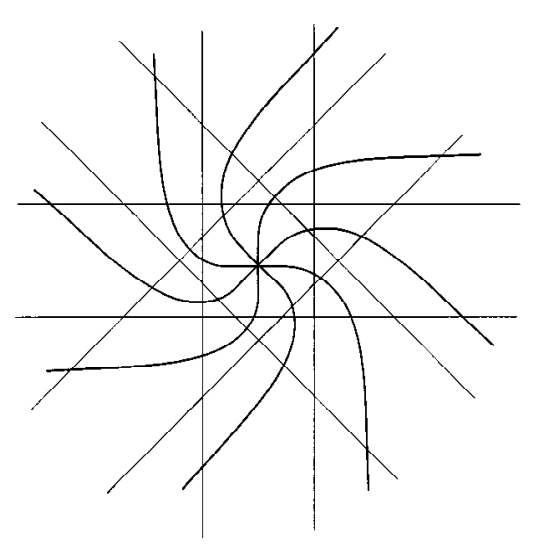

Fig. 5. The arrangement $W_{4}$ with 12 lines and 8 triangles.

For every pair $l_{i}, l_{i+n}$ of parallel lines we construct an additional line $g_{i}$. We lead $g_{1}$ from the unbounded region between the positive end of $l_{1}$ and the negative end of $l_{n}$ to the unbounded region between the positive end of $l_{n+1}$ and the negative end of $l_{2 n}$. The first line crossed by $g_{1}$ is $l_{1}$. Parallel to $l_{n+1}$ line $g_{1}$ crosses $l_{2}, l_{3}, \ldots, l_{n-1}$ and splits quadrangles into two. Before entering $P$ line $g_{1}$ splits the triangle sitting over edge $e_{n}$ into a quadrangle and a triangle. From edge $e_{n}$ line $g_{1}$ joins to point $c$ and then to the opposite edge $e_{2 n}$ to cross lines $l_{2 n}, l_{2 n-1}, \ldots, l_{n+1}$ in this order.

Define lines $g_{2}, \ldots, g_{n}$ by rotational symmetry and note that $g_{1}, \ldots, g_{n}$ all cross in $c$. The arrangement $\mathcal{A} \cup\left\{g_{1}, g_{2}, \ldots, g_{n}\right\}$ has the same number of triangles as $\mathcal{A}$.

So far we still have $n$ pairs of parallel lines. Note however that without increasing the number of triangles we may arbitrarily choose to have the crossing of pair $\left\{l_{i}, l_{i+n}\right\}$ on the side of the positive end of either $l_{i}$ or $l_{i+n}$. Thus $W_{n}$ is itself not just one arrangement, but it represents an exponentially large class of examples.

\section{Triangles in Arrangements of Curves with Multiple Intersections}

In his monograph Grünbaum extends the notion of arrangements in several directions. Let an arrangement of pseudocircles be a family of closed curves with the property that any two curves cross twice. ${ }^{1}$ A digon in such an arrangement is a face bounded by only two of the curves. Grünbaum asks for the relationship between the number of triangles and digons in such arrangements. In particular he conjectures [Gr, Conjecture 3.7] that every digon-free arrangement of pseudocircles contains $2 n-4$ triangles. The only progress on this conjecture is a result of Snoeyink and Hershberger [SH]. They prove $p_{3} \geq 4 n / 3$. The proof is only given for the simple case, i.e., no three curves cross in a single point. However, it is not hard to see that it also applies to the general case.

Based on the arrangements $W_{n}$ from Section 3 it is possible to construct examples of nonsimple arrangements of pseudocircles in $\mathbb{P}$ with only $4 n / 3$ triangles. The idea is to

\footnotetext{
${ }^{1}$ Grünbaum calls this an arrangement of curves.
} 


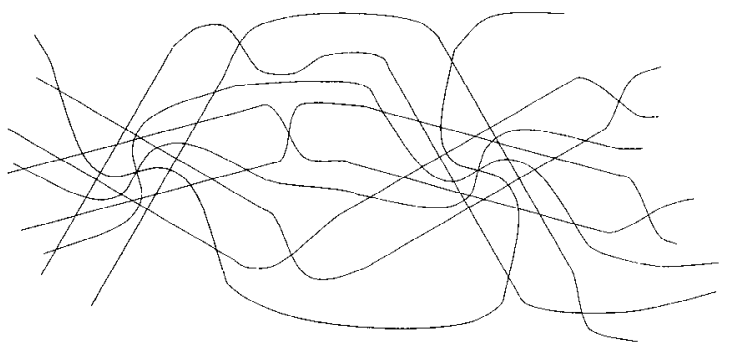

Fig. 6. A digon-free arrangement of 9 two-intersecting curves with 12 triangles.

glue two copies of $W_{n}$ together such that all faces generated by gluing are quadrangles, see Fig. 6. Therefore, the result of Snoeyink and Hershberger is best possible. However, if the arrangement is simple, i.e., no three curves meet in a single point we think that Grünbaum's conjecture should prove correct. For emphasis we restate the conjecture.

Conjecture 1. Every simple digon-free arrangement of pseudocircles contains at least $2 n-4$ triangles.

We feel that the spirit of Euclidean arrangements is captured well with the following generalization. Call an arrangement of $x$-monotone curves with the property that any two curves cross exactly $k$ times a $k$-curve arrangement. Again based on the family $W_{n}$ it is possible to obtain $k$-curve arrangements of $n$ curves with only $2 k n / 3$ triangles. On the other hand we conjecture:

Conjecture 2. Every simple digon-free $k$-curve arrangement contains at least $k(n-2)$ triangles.

If true this would obviously be the best possible since gluing together $k$ appropriate arrangements of pseudolines with $n-2$ triangles each gives arrangements with only $k(n-2)$ triangles.

\section{References}

$\left[\mathrm{BLS}^{+}\right]$A. Björner, M. Las Vergnas, B. Sturmfels, N. White, and G. Ziegler, Oriented Matroids, Cambridge University Press, Cambridge, 1993.

[EP] R. Entringer and G. Purdy, How often is a polygon bounded by three sides? Israel J. Math., 43 (1982), 23-37.

[FP] Z. Füredi and I. Palasti, Arrangements of lines with a large number of triangles, Proc. Amer. Math. Soc., 92 (1984), 561-566.

[FR] D. Forge and J. L. Ramírez-Alfonsín, Straight line arrangements in the real projective plane, Discrete Comput. Geom., 20 (1998), 155-161.

[Go] J. E. Goodman, Proof of a conjecture of Burr, Grünbaum, and Sloane, Discrete Math., 32 (1980), 27-35.

[Gr] B. Grünbaum, Arrangements and Spreads, Regional Conference Series in Mathematics, American Mathematical Society, Providence, RI, 1972. 
[Ha] H. Harborth, Some simple arrangements of pseudolines with a maximum number of triangles, in Discrete Geometry and Convexity, Proc. Conf., New York, 1982, vol. 440, Ann. N.Y. Acad. Sci., 1985, pp. 31-33.

[Kn] D. E. Knuth, Axioms and Hulls, Lecture Notes in Computer Science, vol. 606, Springer-Verlag, Berlin, 1992.

[Le] F. Levi, Die Teilung der projektiven Ebene durch Gerade oder Pseudogerade, in Berichte über die Verhandlungen der sächsischen Akademie der Wissenschafter, Leipzig, Mathematisch-physikalische Klasse 78, 1926, pp. 256-267.

[Pu1] G. Purdy, Triangles in arrangements of lines, Discrete Math., 25 (1979), 157-163.

[Pu2] G. Purdy, Triangles in arrangements of lines, II, Proc. Amer. Math. Soc., 79 (1980), 77-81.

[Ri] G. Ringel, Teilungen der Ebenen durch Geraden oder topologische Geraden., Math. Z., 64 (1956), 79-102.

[Rob] S. Roberts, On the figures formed by the intercepts of a system of straight lines in the plane, and on analogous relations in space of three dimensions, Proc. London Math. Soc., 19 (1889), 405-422.

[Rou1] J.-P. Roudneff, On the number of triangles in simple arrangements of pseudolines in the real projective plane, Discrete Math., 60 (1986), 243-251.

[Rou2] J.-P. Roudneff, Arrangements of lines with a minimum number of triangles are simple, Discrete Comput. Geom., 3 (1988), 97-102.

[Rou3] J.-P. Roudneff, The maximum number of triangles in arrangements of pseudolines, J. Combin. Theory Ser. B, 66 (1996), 44-74.

[Sh] R. W. Shannon, Simplicial cells in arrangements of hyperplanes, Geom. Dedicata, 8 (1979), 179-187.

[SH] J. Snoeyink and J. Hershberger, Sweeping arrangements of curves, in Discrete and Computational Geometry: Papers from the DIMACS Special Year, J. E. Goodman, R. Pollack, and W. Steiger, eds., American Mathematical Society, Providence, RI, 1991, pp. 309-349.

[St] T. Strommer, Triangles in arrangements of lines, J. Combin. Theory Ser. A, 23 (1977), 314-320.

Received February 12, 1998, and in revised form April 7, 1998. 